\title{
SURGICAL MANAGEMENT OF INTRACRANIAL MENINGIOMAS
}

\author{
DR. TARIQ AHNIAD, FCPS (Neurosurgery) \\ Associate Professor \\ Department of Neurosurgery, \\ Punjab Medical College and Allied Hospital, \\ Faisalabad.
}

DR. INAMULLAH ASGHAR, MCPS

Registrar

Department of Neurosurgery,

Punjab Medical College and Allied Hospital, Faisalabad.
DR. AKMAL HUSSAIN, M.S. (Neurosurgery)
Department of Neurosurgery,
Punjab Medical College and Allied Hospital, Faisalabad.

\author{
Dr. Sibef Hasan, MBBS \\ Registrar \\ Department of Neurosurgery, \\ Punjab Medical College and Allied Hospital, \\ Faisalabad. \\ Dr. Habib Sultan, MBBS H \\ Registrar \\ Department of Neurosurgery, \\ Punjab Medical College and Allied Hospital, \\ Faisalabad.
}

\begin{abstract}
Objective: To study the surgical management and outcome of patients having intracranial meningiomas. Design: A retrospective study. Setting: The Department of Neurosurgery Punjab Medical College \& Allied Hospital, Faisalabad. Duration From April 2004 to October 2005. Materials \& Methods: Thirty patients suffering from intracranial meningiomas diagnosed on CT-scan and managed surgically were included in this study. Simpson grade I removal was possible in 21 patients. Grade II removal was done in Three patients while grade 111 removal was possible in four patients, grade IV removal was done in one patient and grade $\mathrm{V}$ in one patient. Post operative XRT was given in cases having grades II, 111, IV and V removal. Follow up was done at six monthly intervals. Outcome was assessed as Good .Fair, and poor depending upon clinical and radiological examinations. RESULTS: Out of 30 patients only one had recurrence after one year of follow up which was removed surgically and postoperative XRT was given. Twenty four patients had good outcome while 5 patients had fair outcome and one had poor outcome. CONCLUSION. Study showed that Simpson grade 1 removal of tumor is still effective treatment in the management of meningiomas. Postoperative XRT is used in Simpson grade 2, 3, 4 and 5 in which gross total removal is not possible due to involvement of neurovascular structures.
\end{abstract}

Key words: Meningioma, Simpson grade, XRT. 


\section{INTRODUCTION}

Meningiomas are the second most common central nervous system tumor, accounting for approximately $20 \%$ of all primary adult intra cranial tumors. The vast majority of meningiomas occur in patients between 50 and 60 years of age, with a twofold higher incidence in women ${ }^{1}$.

Mostly meningiomas are slow growing and as a consequence become quite large before they are detected approximately a quarter of patients with meningioma present with epilepsy. In the remainder the effects of the pressure of the growing tumor (headache, vomiting, etc.) often become evident first. Headache, seizures and focal neurological deficit due to compression of normal brain structures is the normal clinical presentation.

Most common meningioma locations are, Convexity $20 \%$, Sphenoid wing 20\%, olfactory groove $10 \%$, Suprasellar $10 \%$, Posterior fossa) $10 \%$, Intraventricular 2\%, Miscellaneous (e.g., optic nerve, clivius) $3 \%$.

The management goals are to minimize the chance of tumor progression or recurrence and to maintain a low rate of treatment-associated morbidity. Resection of meningiomas has been the treatment of choice since the time of Harvey Gushing ${ }^{2}$.The biological behavior of meningiomas is one of continued growth, ultimately leading to compression of neuronal structures. The treatment of choice is surgery, which is frequently successful in treating these tumors. There are usually two reasons that surgery is ineffective. First, tumor location or proximity to neurovascular structures may make complete resection impossible. Second, the inherent biology of the tumor may give a particular meningioma a greater propensity for recurrence despite seemingly complete resection. Fortunately, histologically atypical or malignant tumors comprise less than $10 \%$ of meningiomas. These two types of tumors are especially disposed to recurrence ${ }^{3}$.It is well established, however, that complete tumor removal is not always possible in keeping with acceptable risk, and tumor recurrence rates range from 18 to $25 \%$ at follow-up periods of 10 years even after complete macroscopic tumor removal has been demonstrated.4i5i6i7To reduce the chance of meningioma recurrence or progression, external beam radiotherapy (EBRT) is commonly performed as a ' postoperative adjunct in patients in whom tumor removal has been subtotal or in whom the meningioma is atypical or malignant. Postoperative EBRT decreases the incidence of tumor recurrence and improves Progression .Free Survival (PFS) after subtotal meningioma resection $^{45^{2} 7^{\prime} 8}$

\section{MATERIAL \& METHODS}

Thirty patients presenting in the Neurosurgical out patient department and also referred patients from other $\sim$ departments of Allied and, DHQ Hospital Faisalabad were included in this study. Patients were provisionally diagnosed on CT-scan [control and contrast] as cases of meningiomas. Twenty one patients were operated with craniotomy and total excision of tumor with involved dura and overlying bone if any [Simpson grade I] .Resected tumors were sent for histopathological examination and diagnosis was confirmed. The cranial defect in 20 patients was small and was located in temporal and parietal area and did not require cranioplasty while in one patient there was a large frontal bone defect which was repaired with autologous bone. Nine patients in which tumor was adherent with neurovascular structures like dural venous sinuses and cranial nerves subtotal resection [Simpson grade II, III, IV and V] was done. These cases underwent postoperative XRT. Out come was assessed as;

Good, free of major neurological deficit and able to return to previous activity level.

Fair, independent but not able to return to full 
activity because of new neurological deficit or significant preoperative deficit that did not fully recover.

Poor, dependent. .

All patients underwent follow up at six monthly intervals with check CT-scan [control and contrast].

\section{RESULTS}

Thirty patients of meningiomas undergoing surgical management were included in the study admitted from April 2004 to October 2005. Among thirty patients 20 were females and 10 were male [Fig $1,2]$ ranging in age

from 25 to $\sim 65$ years [Table I],

\begin{tabular}{|c|c|}
\hline \multicolumn{2}{|c|}{ Table-1. Grade distribution. } \\
\hline Grade (Simpson) & No. Of patients \\
\hline Grade 1 & 3 \\
\hline Grade II & 4 \\
\hline Grade III & 1 \\
\hline Grade IV & \\
\hline Grade V & \\
\hline Fig-1. Sex distribution \\
\hline
\end{tabular}

Among 30 patients, 16 were cases of convexity meningiomas, 8 were Parafalcine, 5 were sphenoid wing and one was a tentorial meningioma.

Out of 16 patients of convexity meningiomas, 3 cases were of recurrent meningiomas. Fifteen patients were operated with Simpson grade 1 One had subtotal removal [Simpson grade 3] having a large tumor who under went XRT in postop.period.XRT was also given in recurrent cases. Three patients had significant temporary worsening of hemiparesis and sensory loss, one with difficulty in controlling seizures, one with wound infection and one with DVT. Among 16 patients 13 had good outcome 2 had fair outcome and only one had poor outcome. There has been evidence of only one recurrence at one year follow up. This was in a patient in which Simpson gradeS removal was done which later on was surgically removed.

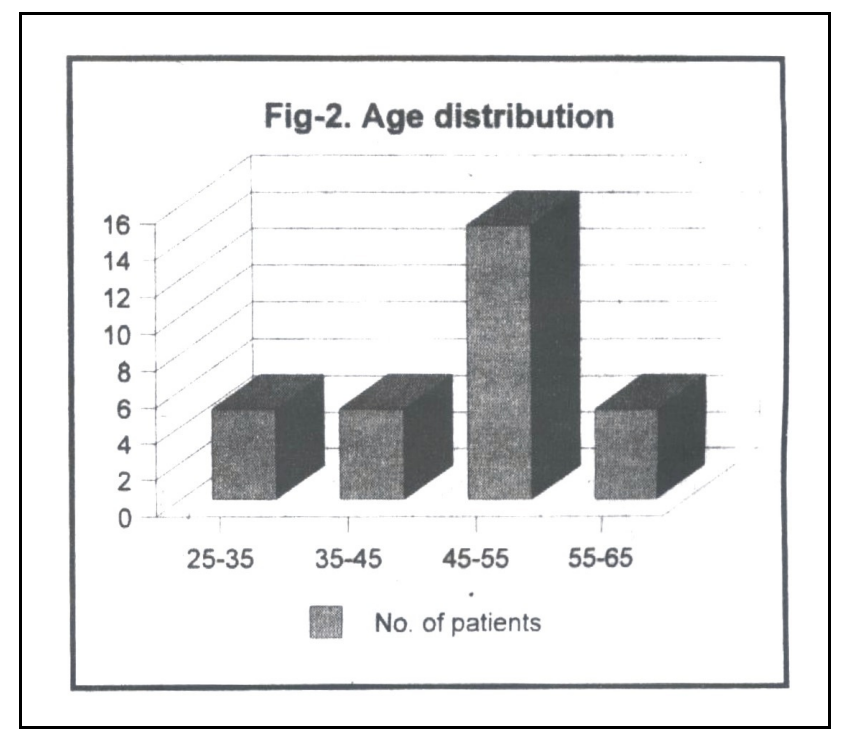

Among eight patients of parafalcine meningiomas five were operated with Simpson gradel and three had subtotal removal [Simpson gradeS] because of involvement of superior saggital sinus who under went post op. XRT.Two patients had postoperatively worsening of neurological deficits which improved over weeks. There has been no 
evidence of recurrence in any patient including patients with subtotal removal.
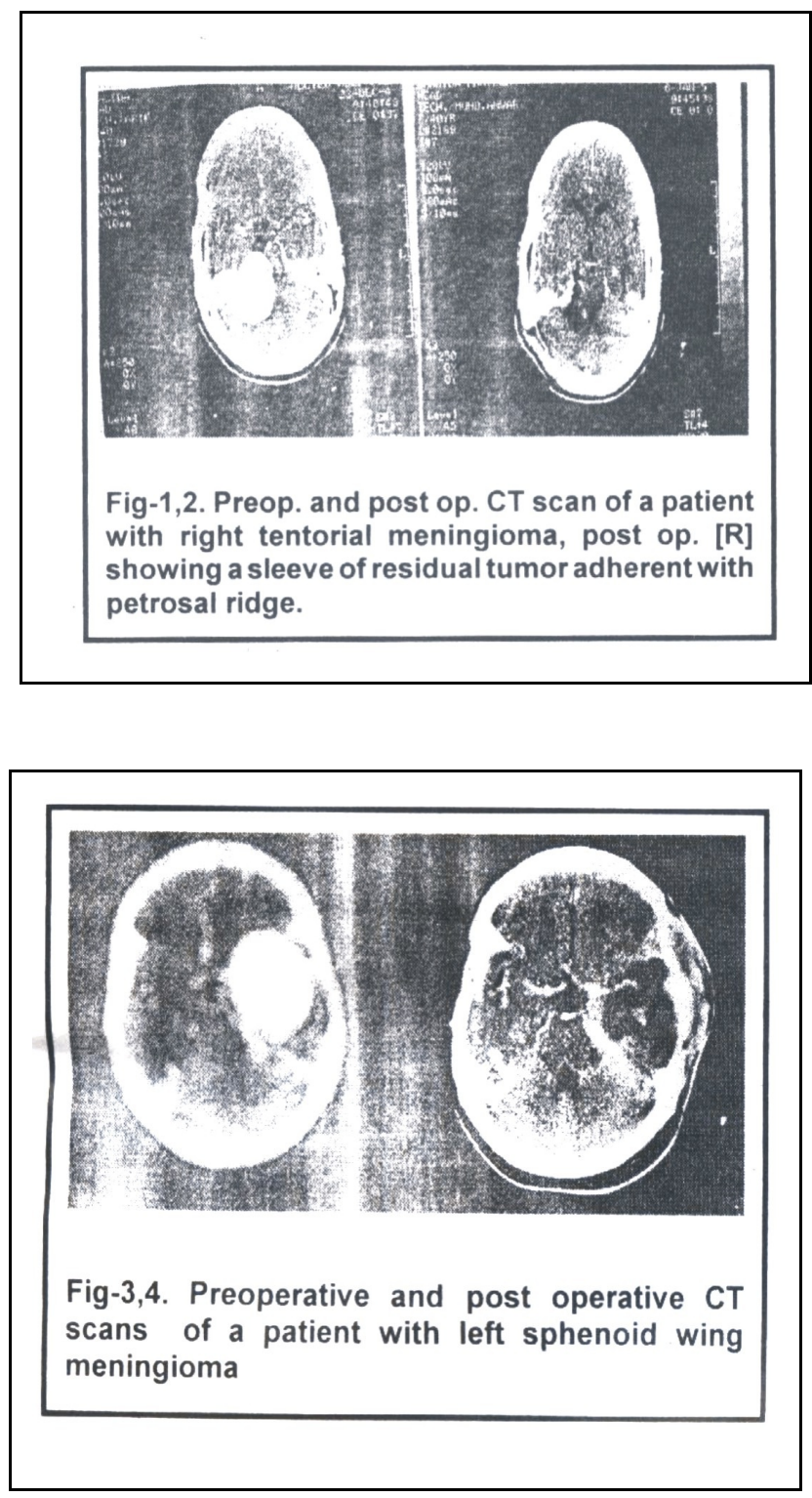

Out of five patients of sphenoid wing meningiomas, four patients were operated with Simpson grade 2 and rest of patients were operated with Simpson grade 3, all under went postoperative XRT There were no post op. complications and all returned to full activity. There has been no recurrence at six months and one year of follow up. Brotchi and
Bonnel reported same results with nine patients ${ }^{9}$.

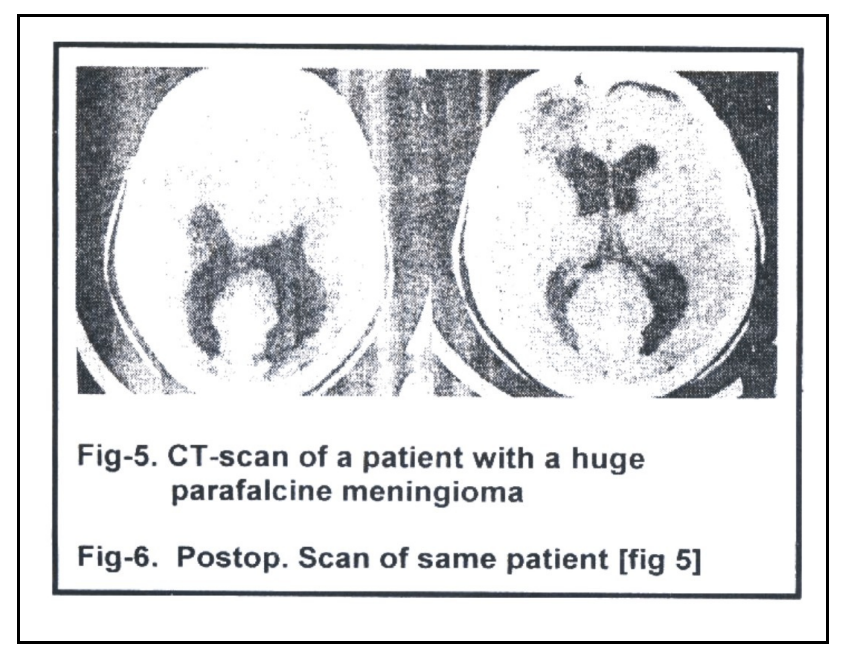

One patient with posterior fossa meningioma involving posterior/lateral tentorium underwent subtotal removal [Simpson gradeS] with postop. XRT.Subtotal removal was done due to the involvement of transverse sinus. Follow up at one year showed no recurrence.

Sugita and Suzuki10 reported on 49 patients operated upon for tentorial meningiomas with $88 \%$ excellent or good results. The operative mortality was $4 \%$.

\section{DISCUSSION}

For more than 50 years, tumor excision has been the treatment of choice for patients with intracranial meningiomas. The paper published by Simpson 11 in $1957 \ldots$ showed the correlation between the degree of meningioma resection and the risk of recurrence. He noted a rate of symptomatic tumor recurrence of 9,19 , and $29 \%$ in patients who underwent Grades 1,2 , and 3 resections, respectively. Based on these findings, the basic tenet of meningioma management became surgical treatment should be aimed at complete tumor removal as well as the adjacent dura and involved bone[Simpson grade] Improved anesthetic, neuroimaging, and 
microsurgical techniques have increased the number of patients in whom complete resection of meningiomas has been possible in conjunction with acceptable morbidity. Some meningiomas, however, invade adjacent neurovascular structures and simply cannot be completely excised without posing unacceptable risk. ${ }^{12}{ }^{13 \ln }$ our series of 30 patients Simpson's grade I removal was possible in $21(70 \%)$ patients while 15 of these 21 patients were of convexity. Investigators of recent series on meningioma surgery have reported that gross-total resection is possible in 38 to $100 \%$ of patients, depending primarily on tumor location ${ }^{14^{\prime} 1516^{\prime} 17^{\prime} 18}$ Even when a gross-total resection has been achieved, however, tumor recurrence rates can range from 18 to $25 \%$ at 10 years ${ }^{4^{\prime} 6^{\prime} 7^{\prime} 19 !}$ The impact of subtotal resection (Simpson grade $>/=3$ ) on this population is even more significant. Jung, et $\mathrm{al}^{20}$ reported a 5 -year tumor progression rate of $40 \%$ in patients in whom petroclival meningiomas were subtotally resected. The rate of tumor growth in that series was $4 \mathrm{~mm}$ annually. Klink, et al., 21 noted that $18(62 \%)$ of 29 patients undergoing "nonradical" surgery of parasellar and cavernous sinus meningiomas suffered tumor recurrence/progression within a mean follow-up interval of 13.6 years. The tumor progression rate in patients followed more than 15 years was $89 \%$ (eight of nine patients). Kallio, et al 22 found the relative risk of death due to subtotal resection was nearly equal (4.2-fold greater) to the risk in patients with atypical or malignant meningiomas in their review of 935 patients

To reduce the chance of meningioma recurrence or progression, EBRT is frequently used as a postoperative adjunct in patients with subtotally excised tumors or in those whose tumor displayed either atypical or malignant features. In our study EBRT was given in ten patients. ${ }^{\wedge}$ TMumerous studies have documented that postoperative radiotherapy decreases the incidence of tumor recurrence and improves survival for patients after subtotal meningioma resection ${ }^{4,5,7,8}$ The same result?

were found in our study. Moreover, selected patients have been managed with EBRT alone if they were considered medically unfit for surgery or if their tumor was deemed unresectable.

Over the past two decades, radio surgery has been conducted as an alternative to surgery and EBRT in patients with meningiomas who have symptoms from mass effect. In a paper on patients with benign $\wedge$ meningiomas, Kondziolka, et $\mathrm{al}^{23}$ reported that $91 \%$ remained neurologically stable at 5 - to 10 -year follow-up after radio surgery. Therefore, radio surgery performed as a primary procedure may produce better tumor control rates than after previous surgery.

Chemotherapy is occasionally used to treat meningioma.Hormonal blocking agents and interfergn also may be used

MIB-1 labeling index staining is a good predictor for refractory meningiomas.

Indicators of outcome in Meningioma surgery are age, tumour location and accessibility and extent of removal.

Treatment options for recurrence or incomplete resection include further surgery, conventional external beam irradiation, stereotactic radio surgery, and systemic therapies ${ }^{824}$ - Most patients with malignant meningiomas will receive radiation therapy after surgery ${ }^{25} 26$ The most promising antineoplastic agent, hydroxyurea, has proven to be an effective treatment for recurrent or unresectable meningiomas ${ }^{272829}$ Hormonal therapy has been attempted using agents that block estrogen or progesterone receptors, buttrials with tamoxifen, medroxyprogesterone acetate, megestrol acetate, and the progesterone receptor antagonist RU486 also have been disappointing ${ }^{30}$. In our study there 
were three cases of recurrent meningioma which were pre-operated and post operative XRT was given.

In spite of the advances made over the past two decades in neuro-anaesthesia, neuro radiology and neurosurgical microsurgery, meningiomas of the base of the skull are still a challenge in the field of neurosurgery and needs further research.

\section{CONCLUSION}

Aggressive surgical resection [Simpson gradel] continues to be the mainstay of current meningioma management, with advances in microsurgical techniques facilitating the safety and effectiveness of this approach. Complete surgical removal of meningiomas provides the optimal opportunity of long term remission. Advances in skull base surgical approaches have greatly improved patient outcomes for tumors in precarious locations once thought to be inoperable. Complete surgical resection is often curative. For most incompletely resected or recurrent tumors not previously irradiated, radiotherapy is administered. Radiotherapy may be administered as either conventional external beam irradiation or stereotactically by linear accelerator, gamma knife or cyber knife radio surgery. When the meningioma is unresectable or all other treatments (e.g., surgery and radiotherapy) have failed, hormonal chemotherapy may be considered. Notwithstanding limited data, hydroxyurea has been modestly successful in patients with recurrent meningiomas.

The explosive growth in the number of meningioma patients selecting radio surgery in recent years attests to the fact that the goal of controlling meningioma growth while preserving neurological function is very attractive to patients. The use of radio surgery as the primary treatment of smaller meningiomas, and its use in combination with surgical decompression or subtotal removal (with avoidance of neurological deficits that could arise from more radical removal) in the treatment of larger tumors, is emerging as the standard of care at many centers.

\section{REFERENCES}

1. Carroll RS, Zhang J, Black PM. Expression of estrogen receptors alpha and beta in human meningiomas. J Neurooncol. 1999;42:109-116.

2. Gushing H, Eisenhardt L: Meningiomas: Their Classification, Regional Behavior, Life History, and Surgical End Results. Springfield, IL: Charles C Thomas, 1938

3. Gonzales MF. Classification of brain tumors. In: Kaye AH, Laws ER, eds. Brain Tumors: An Encyclopedic !»,, Approach. Edinburgh, New York: Churchill Livingstone; 1995:675.

4. Barbara NM, Gutin PH, Wilson CB, et al: Radiation therapy in the treatment of partially resected meningiomas. Neurosurgery 20:525 528,1987

5. Condra KS, Buatti JM, Mendenhall WM, et al: Benign meningiomas: primary treatment selection affects survival. Int J Radiat Oncol Biol Phys 39:427 436,1997

6. Mathiesen $\mathrm{T}$, Lindquist $\mathrm{C}$, Kihlstrom $\mathrm{L}$, et al: Recurrence of cranial base meningiomas. Neurosurgery 39:2 9, 1996.

7. Mirimanoff RO, Dosoretz DE, Linggood RM, et al: Meningioma: analysis of recurrence and progression following neurosurgical resection. J Neurosurg 62:18 24,1985

8. Goldsmith BJ, Wara WM, Wilson CB, etai: Postoperative irradiation for subtotally resected meningiomas. A retrospective analysis of 140 patients treated from 1967 to 1990 . J Neurosurg 80:195 201,1994

9. Brotchi, J., and Bonnal, J. P. Lateral and middle sphenoid wing meningiomas. In: Meningiomas, edited by 0. AI-Mefty, pp. 413-425. Raven Press, New York, 1991.

10. Steiner, L., Lindquist, C., and Steiner, M. Meningiomas and gamma knife radiosurgery. In: Meningiomas, edited by 0. AI-Mefty, pp. 263-272. 
Raven Press, New York, 1991.73. Sugita, K., and Suzuki, Y. Tentorial meningiomas. In: Menningiomas, edited by 0. AI-Mefty, pp. 357-361. Raven Press, New York, 1991.

11. Simpson D: The recurrence of intracranial meningiomas after surgical treatment. J Neural Neurosurg Psychiatry 20:22 39,1957

12. Kotapka MJ, Kalia KK, Martinez AJ, et al: Infiltration of the carotid artery by cavernous sinus meningioma. J Neurosurg 81:252 255,1994

13. Larson JJ, van Loveren HR, BalkoMG.etal: Evidence of meningioma infiltration into cranial nerves: clinical implications for cavernous sinus meningiomas. J Neurosurg 83:596 599, 1995

14. Abdel Aziz KM, Sanan A, van Loveren HR, et al: Petroclival meningiomas: predictive parameters for transpetrosal approaches. Neurosurgery 47:139 152, 2000

15. Arnautovic K1, AI-Mefty 0: Primary meningiomas of the «s jugular fossa. J Neurosurg 97:12 20, 2002

16. Couldwell WT, Fukushima T, Giannotta SL, et al: Petroclival meningiomas: surgical experience in 109 cases. J Neurosurg 84:20 28,1996

17. De Jesus 0, Sekhar LN, Parikh HK, et al: Long-term follow-up of patients with meningiomas involving the cavernous sinus: recurrence, progression, and quality of life. Neurosurgery 39: 915920,1996

18. DeMonte F, Smith HK, AI-Metty 0: Outcome of aggressive removal of cavernous sinus meningiomas. J Neurosurg 81: 245 251, 1994

19. Chang SD, Adler JR Jr: Treatment of cranial base meningiomas with linear accelerator radiosurgery. Neurosurgery 41: 10191027,1997

20. Jung H, Yoo H, Paek SH, et al: Long-term outcome and growth rate of subtotally resected petroclival meningiomas: experience with 38 cases. Neurosurgery $46: 567575,2000$
21. Klink DF, Sampath P, Miller NR, et al: Long-term visual outcome after nonradical microsurgery patients with parasellar and cavernous sinus meningiomas. Neurosurgery 47:24 32, 2000

22. Kallio M, Sankila R, Hakulinen T, et al: Factors affecting operative and excess long-term mortality in 935 patients with intracranial meningioma. Neurosurgery 31:212,1992

23. Kondziolka D, Levy El, Niranjan A, et al: Long-term outcomes after meningioma radiosurgery: physician and patient perspectives. J Neurosurg 91:44 50,1999

24. Rosenthal MA, Ashley DL, Cher L. Treatment of high risk or recurrent meningiomas with hydroxyurea. J Clin

Neurosci. 2002;9:156-158. 28.

25. Jaaskelainen J, Haltia M, Servo A. A typical and anaplastic meningiomas: radiology, surgery, radiotherapy, and outcome. Surg Neural. 1986;25:233-242. 29.

26. Hug EB, Devries AJhornton AF, et al. Management of atypical and malignant meningiomas: role of highdose, 3D-conformal radiation therapy. J Neurooncol. 2000;48:151-160. 30 .

27. Rosenthal MA, Ashley DL, Cher L. Treatment of high risk or recurrent meningiomas with hydroxyurea. J Clin Neurosci. 2002;9: 156-158.

28. Schrell DM, Rittig MG, Anders M. Hydroxyurea for treatment of unresectable and recurrent meningiomas. II. Decrease in the size of meningiomas in patients treated $\mathrm{Wj}$ th hydroxyurea. J Neurosurg. 1997;86:840-844.

29. Mason WP, Gentili F, Macdonald DR, etal. Stabilization of dis-ease progression by hydroxyurea in patients with recurrent or unresectable meningioma. J Neurosurg. 2002;97:341-346.

30. Blankenstein MA, Verheijen FM, Jacobs JM, et al. Occurrence, regulation, and significance of progesterone receptors in human meningioma. Steroids. 2000;65:795-800. 